\title{
Impact of Channel Asymmetry on Base Station Cooperative Transmission with Limited Feedback
}

\author{
Xueying Hou, and Chenyang Yang \\ School of Electronics and Information Engineering, Beihang University, Beijing, China
}

\begin{abstract}
Base station (BS) cooperative transmission, also known as coordinated multi-point transmission (CoMP), is an effective way to avoid inter-cell interference in universal frequency reuse cellular systems. To gain the promised benefit, however, huge feedback overhead is in demand to gather the channel information. In this paper, we analyze the impact of channel asymmetry, which is inherent in CoMP systems, on downlink BS cooperative transmission with limited feedback. We analyze the per-user rate loss of a multi-user CoMP system led by quantization. Per-cell quantization of multicell channels is considered, which quantizes the local channel and cross channel separately and is more feasible in practice. From both the analytical and simulation results, we provide a whole picture on various critical factors that lead to the performance loss. Specifically, we show that the per user rate loss led by limited feedback depends on the location of its paired users, except for relying on its own signal to noise ratio and the quantization errors as in single cell multi-user multiple antenna systems. This implies that the quantization accuracy required for local and cross channel of each user depends on the locations of its own as well as its paired users.
\end{abstract}

\section{INTRODUCTION}

Base station (BS) cooperative transmission, which is also known as coordinated multi-point transmission (CoMP), is an effective strategy to mitigate the inter-cell interference (ICI) arisen from universal frequency reuse cellular systems. As a promising transmit strategy, coherent cooperative transmission can enhance the downlink spectrum efficiency by using multiuser (MU) multiple-input multiple-output (MIMO) precoding [1-3], when both data and channel state information (CSI) can be sent to a central unit (CU) via backhaul links.

For frequency division duplexing (FDD) MU-MIMO systems, the required CSI at the transmitter can be obtained through uplink feedback using limited number of bits [4]. The impact of limited feedback on the performance of single cell MU-MIMO transmission under spatial independent channels has been investigated in [5] and [6]. It shows that the quantization error of limited feedback leads to a throughput ceiling at high signal-to-noise ratio (SNR) level [5]. In order to achieve full multiplexing gain, the feedback bits per mobile station (MS) should increase linearly with SNR at a slope proportional to the number of transmit antennas.

Despite that the considered CoMP system with the CU can be viewed as a large MIMO system with a "super BS", there are distinct differences between the channels of the single cell MIMO transmission and CoMP. An inherent feature of CoMP channels is asymmetry, which means that the average channel energy from different BSs to one MS are different. This leads to different performance when the same transmit strategy is applied in the two systems [7]. It also provides new opportunities for developing new transmit strategies [8]. For single user (SU) MIMO CoMP, dynamic allocating the feedback bits among the channels with different energies is shown to perform better than the equally bits allocation through simulations [9], and a per-cell bit allocation algorithm based on user location is provided aiming to achieve a normalized SNR upper bound [10]. For coordinated beamforming in multicell multiuser system, the CSI quantization and codebook design are studied in [11] and [12], where only CSI are shared among the BSs thereby the addressed scheme is not the MU-MIMO CoMP. In [11], an adaptive feedback bits assignment among desired and interfering channels exploiting the difference of their average energies is proposed, where the desired and the interfering CSI are quantized using separate codebooks. A better CSI quantization method is proposed in [12], which quantizes the concatenation of the desired and interfering channel vectors by choosing a codeword from a designed codebook.

In this paper, we analyze the impact of channel asymmetry on MU-MIMO CoMP using multicell zero-forcing beamforming (ZFBF) with limited feedback. We consider per-cell channel quantization since it is of practical importance, which respectively quantizes the channel direction information (CDI) of local channel, i.e., the channel between the BS and the mobile station (MS) who are in the same cell, and that of cross channels, i.e., the channels between the BS and the MS who are in different cells. We analyze the rate loss of MUMIMO CoMP caused by limited feedback. We will show that the rate loss not only depends on those factors dominating the rate loss of single cell MU-MIMO, which include the CSI quantization error, the number of transmit antennas and the receive SNR, but also relies on the location of the paired MSs. The performance loss of a MS led by limited feedback reduces significantly when its paired MSs locate near their serving BSs. This is especially true when this MS does not locate at the cell edge.

Notations: Boldface upper case letters $\mathbf{X}$ represent matrices, boldface lower case letters $\mathbf{x}$ denote vectors and standard lower case letters $x$ denote scalars. $\mathbf{X}(:, i)$ represents the $i$ th column of $\mathbf{X}$. The transpose and Hermitian conjugate transpose of $\mathbf{X}$ are denoted as $\mathbf{X}^{T}$ and $\mathbf{X}^{H}$, respectively. $\mathbb{E}\{x\}$ is the expectation of the random variable $x .|x|$ represents the norm 


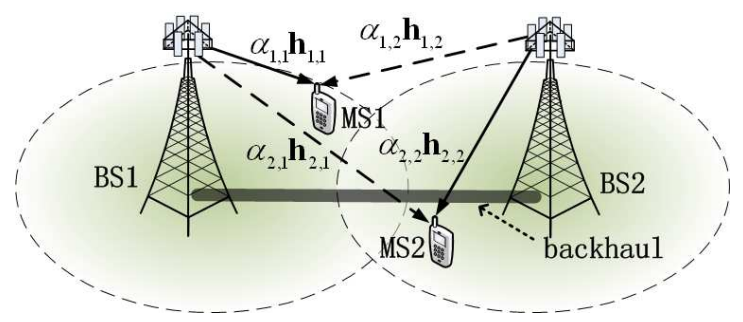

Fig. 1. An example of CoMP system with two multiple antenna BSs cooperatively serving two single antenna MSs. The solid lines denote the local channel while the dash lines represent the cross channels.

of the complex scalar $x$, and $\|\mathbf{x}\|$ represents the two-norm of $\mathrm{x}$.

\section{SySTEM MODEL}

Consider a cellular system with $N$ cells, each contains one BS equipped with $n_{t}$ antennas. To highlight the impact of channel asymmetry on the performance of MU-MIMO CoMP, we assume that only one single antenna MS will be selected from each cell for MU-MIMO CoMP. When CSI from the $N$ BSs to the $N$ MSs are available in the CU, MU-MIMO precoding is used for coherent cooperative transmission. An example of CoMP system is shown in Fig 1 where either BS 1 or BS 2 can serve as the CU.

The global composite channel vector from all cooperative $\mathrm{BSs}$ to the $k$ th MS is represented by

$$
\mathbf{g}_{k}=\left[\alpha_{k, 1} \mathbf{h}_{k, 1}, \cdots, \alpha_{k, N} \mathbf{h}_{k, N}\right]
$$

where $\alpha_{k, b}$ and $\mathbf{h}_{k, b} \in \mathbb{C}^{1 \times n_{t}}$ are respectively the large scale fading factor and the small scale fading channel vector from the $b$ th $\mathrm{BS}$ to the $k$ th $\mathrm{MS}, \alpha_{k, b}$ includes path loss and shadowing, $\alpha_{k, b}^{2}$ is the average energy of the composite channel $\alpha_{k, b} \mathbf{h}_{k, b}$. For MS $k$ served by BS $k, \alpha_{k, k} \mathbf{h}_{k, k}$ is its composite local channel whereas $\alpha_{k, b} \mathbf{h}_{k, b}$ for $b \neq k$ are its composite cross channels.

Let the data transmitting to all $N$ MSs be $\mathbf{d}=$ $\left[d_{1}, \cdots, d_{N}\right]^{T}$. Without loss of generality, we assume that $\mathbb{E}\left\{\mathbf{d d}^{T}\right\}=\mathbf{I}$. The received signal at MS $k$ is

$$
y_{k}=\sqrt{P} \mathbf{g}_{k} \mathbf{v}_{k} d_{k}+\sqrt{P} \sum_{j=1, j \neq k}^{N} \mathbf{g}_{k} \mathbf{v}_{j} d_{j}+n_{k}
$$

where $P$ is the transmit power for each MS, $\mathbf{v}_{j}$ is a $N n_{t} \times 1$ vector representing a unitary precoding vector from all cooperative BSs for $\mathrm{MS} j$, and $n_{k}$ is additive white Gaussian noise (AWGN) with zero mean and variance $\sigma_{n}^{2}$.

Then we have the signal to noise plus interference ratio (SINR) at MS $k$ as

$$
\mathrm{SINR}_{k}=\frac{P\left|\mathbf{g}_{k} \mathbf{v}_{k}\right|^{2}}{\sigma_{n}^{2}+P \sum_{j=1, j \neq k}^{N}\left|\mathbf{g}_{k} \mathbf{v}_{j}\right|^{2}},
$$

and the average throughput of MS $k$ as

$$
R_{k}=\mathbb{E}_{\mathbf{H}, \mathbf{V}}\left\{\log _{2}\left(1+\mathrm{SINR}_{k}\right)\right\} .
$$

\section{A. Finite Rate Feedback Model}

We assume that MS $k$ has perfect knowledge of the global channel vector $\mathbf{g}_{k}$. We consider per-cell quantization to quantize $\mathbf{g}_{k}$, i.e., MS $k$ separately quantizes the channel vector $\alpha_{k, b} \mathbf{h}_{k, b}$ between MS $k$ and BS $b, b=1, \cdots, N$. After MS $k$ feeds back these quantized per-cell CSI, BS $k$ uses these feedback to reconstruct the global channel of MS $k$ and then sends it to the CU.

Denote the instantaneous norm and CDI of the global channel between MS $k$ and BS $b$ as $\rho_{k, b}=\alpha_{k, b}\left\|\mathbf{h}_{k, b}\right\|$ and $\overline{\mathbf{h}}_{k, b}=\mathbf{h}_{k, b} /\left\|\mathbf{h}_{k, b}\right\|$, where $\rho_{k, b}$ includes both large scale fading factor and small scale fading channel norm. To highlight the effect of CDI quantization, we assume that the channel norm $\rho_{k, b}, b=1, \cdots, N$, are directly fed back without quantization as in [5, 6, 11, 12]. Denote the codebook for quantizing the CSI between MS $k$ and BS $b$ as $\mathcal{C}_{k, b}$, which is consist of unit norm row vectors $\mathbf{c}_{i}, i=1, \cdots, 2^{B_{k, b}}$. The quantized CDI $\hat{\mathbf{h}}_{k, b}$ is set to be $\mathbf{c}_{i_{k, b}}$ with the index $i_{k, b}$ chosen as follows

$$
i_{k, b}=\arg \max _{1 \leq j \leq 2^{B} k, b}\left|\overline{\mathbf{h}}_{k, b} \mathbf{c}_{j}^{H}\right|^{2} .
$$

The quantization error is defined as $\sin ^{2} \theta_{k, b}=1-\left|\overline{\mathbf{h}}_{k, b} \hat{\mathbf{h}}_{k, b}^{H}\right|^{2}$.

After MS $k$ quantizes the CDI for both local and cross channels, it feeds back the index $\left\{i_{k, 1}, \cdots, i_{k, B}\right\}$ to its serving $\mathrm{BS}$, i.e., the $\mathrm{BS} k$, which requires $\sum_{b=1}^{N} B_{k, b}$ bits. Based on the index and the codebook, BS $k$ reconstructs the global channel of MS $k$ as follows,

$$
\hat{\mathbf{g}}_{k}=\left[\rho_{k, 1} \hat{\mathbf{h}}_{k, 1}, \cdots, \rho_{k, N} \hat{\mathbf{h}}_{k, N}\right]
$$

When all $N$ BSs obtained the reconstructed global channel vectors, they send the quantized channel vectors to the $\mathrm{CU}$ via a low latency and error free backhaul.

\section{B. Multicell Zero-forcing Beamforming}

After the reconstructed global channel vectors from $N$ MSs to $N$ BSs are available at the $\mathrm{CU}$, a multicell $\mathrm{ZFBF}$ is obtained as follows

$$
\mathbf{V}=\hat{\mathbf{H}}^{H}\left(\hat{\mathbf{H}} \hat{\mathbf{H}}^{H}\right)^{-1}
$$

where $\hat{\mathbf{H}}=\left[\hat{\mathbf{g}}_{1}^{H}, \cdots, \hat{\mathbf{g}}_{N}^{H}\right]^{H}$. We consider per-user power constraint as in [5]. Then the beamforming vector of all cooperative BSs for the $k$ th $\mathrm{MS}$ is obtained by normalizing the $k$ th column of $\mathbf{V}$ as $\mathbf{v}_{k}=\mathbf{V}(:, k) /\|\mathbf{V}(:, k)\|$.

\section{RATE LOSS ANALYSIS OF COMP TRANSMISSION}

Similar to [5, 6], we study the rate loss led by the limited feedback which can be bounded by [5],

$$
\Delta R_{k}<\log _{2}\left[1+\frac{P}{\sigma_{n}^{2}} \mathbb{E}\left\{\sum_{j \neq k}\left|\mathbf{g}_{k} \mathbf{v}_{j}\right|^{2}\right\}\right] .
$$

The rate loss caused by limited feedback in single cell MU-MIMO systems is well analyzed in [5]. Under the assumption that both the channel vector and its quantization are isotropic distributed, which holds for spatial independent fading channels and the channels are quantized by random 
vector quantization, [5] shows that the rate loss is dominated by the quantization error, the number of transmit antennas and the receive SNR. When the quantization error is fixed, the rate loss will increase with SNR.

In CoMP systems, due to the inherent asymmetry feature of multicell channels, the CDI distribution of the global channel is largely dependent on the large scale fading factors. As a result, the CDI distribution of the global channel is no longer isotropic. In the following, we will show that the rate loss of a MS in MU-MIMO CoMP systems not only relies on those factors which dominate the per MS rate loss in single cell MU-MIMO systems, but also depends on the location of its paired MSs. Contrary to the conclusion drawn in single cell MU-MIMO systems [5], the rate loss in MU-MIMO CoMP systems may even decrease with the increase of SNR.

To decouple the impact of limited feedback on scheduling and precoding, we assume that MU MIMO scheduling is conducted before the downlink CoMP based on the quantized global channels. We can use many scheduling algorithms for pairing the multiple users, e.g., the semi-orthogonal user selection (SUS) [13], which chooses MSs that have high channel qualities and are mutually semi-orthogonal. Specifically, the correlation of the quantized channels between the scheduled MS $k$ and MS $j$, which is defined as $\frac{\left|\hat{\mathbf{g}}_{k} \hat{\mathbf{g}}_{j}^{H}\right|}{\left\|\hat{\mathbf{g}}_{k}\right\|\left|\hat{\mathbf{g}}_{j}\right|}$, should be less than a threshold.

The ZFBF consists of pseudo-inverse of the channel matrix, which is rather involved for analysis. For mathematical tractability, we assume that the selected MSs are mutually orthogonal in terms of their quantized globe channel directions, i.e., we set the threshold in SUS as 0. In practice, this assumption holds when the number of MSs is large enough. Although this assumption is too strong, we will verify through simulation results in section IV that the following analysis is applicable for the realistic cellular scenario without the assumption.

Under this assumption, the precoder vector for the MS $j$ reduces to $\mathbf{v}_{j}=\hat{\mathbf{g}}_{j}^{H} /\left\|\hat{\mathbf{g}}_{j}\right\|$. Then the rate loss in (7) can be expressed as

$$
\Delta R_{k}<\log _{2}\left[1+\frac{P}{\sigma_{n}^{2}} \mathbb{E}\left\{\sum_{j \neq k} \frac{1}{\left\|\hat{\mathbf{g}}_{j}\right\|^{2}}\left|\mathbf{g}_{k} \hat{\mathbf{g}}_{j}^{H}\right|^{2}\right\}\right] .
$$

Substituting the expressions of the global channel vector $\mathrm{g}_{k}$ and its per-cell quantization $\hat{\mathrm{g}}_{k}$ into (8) and after some manipulations, we can obtain the rate loss of MS $k$ as (see Appendix $\mathrm{A}$ for details),

$$
\Delta R_{k}<\log _{2}[1+\frac{n_{t}}{n_{t}-1} \sum_{j \neq k} \underbrace{\sum_{b=1}^{N} \beta_{j, b} \gamma_{k, b}^{2} \sin ^{2} \theta_{k, b}}_{I_{j}}]
$$

where $\beta_{j, b}=\frac{\alpha_{j, b}^{2}}{\sum_{b=1}^{N} \alpha_{j, b}^{2}}$ only depends on the locations of its paired users, $\gamma_{k, b}^{2}=\frac{P \alpha_{k, b}^{2}}{\sigma_{n}^{2}}$ is the receive SNR of the signal from BS $b$ to MS $k, \sin ^{2} \theta_{k, b}$ is the per-cell quantization error of $\overline{\mathbf{h}}_{k, b}$.

The term $I_{j}$ in (9) represents the inter-user interference from

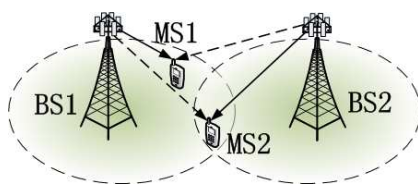

(a) MS2 locates at cell edge

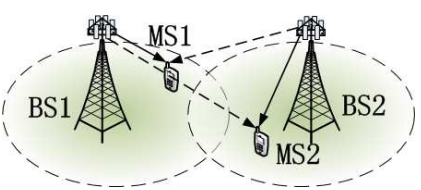

(b) MS2 locates at cell center
Fig. 2. A CoMP system with two BSs cooperatively serving two MSs.

MS $j$ to MS $k$. We can find that $I_{j}$ not only depends on the channel quantization error, the number of transmit antennas at each BS and the receive SNR of MS $k$, but also relies on the value of $\beta_{j, b}$. This indicates that the rate loss of MS $k$ is closely related to the positions of its paired MSs.

In order to provide a more clear picture on how the multicell MS pairing will affect the rate loss, we consider a simple but fundamental scenario where two BSs with multiple antennas cooperatively serve two single-antenna MSs, as shown in Fig 2 Without loss of generality, we analyze the rate loss of MS 1, which is upper bounded by

$$
\begin{aligned}
\Delta R_{1} & <\log _{2}\left[1+\frac{n_{t}}{n_{t}-1}\left(\beta_{2,1} \gamma_{1,1}^{2} \sin ^{2} \theta_{1,1}+\beta_{2,2} \gamma_{1,2}^{2} \sin ^{2} \theta_{1,2}\right)\right] \\
& \triangleq \Delta R_{1 u b}
\end{aligned}
$$

where $\beta_{2,1}=\frac{1}{1+\alpha_{2,2}^{2} / \alpha_{2,1}^{2}}$ and $\beta_{2,2}=\frac{1}{1+\alpha_{2,1}^{2} / \alpha_{2,2}^{2}}$.

The value of $\gamma_{1,1}^{2}$ and $\gamma_{1,2}^{2}$ respectively represent the local and cross link receive SNR of MS1, and $\sin ^{2} \theta_{1,1}$ and $\sin ^{2} \theta_{1,2}$ are the quantization errors of the local CDI and the cross CDI. The values of $\beta_{2,1}$ and $\beta_{2,2}$ depend on the average channel energy ratio of the local channel and cross channel of MS2, i.e. $\alpha_{2,2}^{2} / \alpha_{2,1}^{2}$. Here, MS2 is the paired MS for MS1. To observe the impact of MS2 on the performance of MS1, we analyze the following two cases when MS2 locates at cell edge and at cell center.

\section{A. When MS2 Locates at Cell Edge}

As shown in Fig 2 (a), when MS 2 locates at the edge of cell 2, we have $\alpha_{2,1}^{2}=\alpha_{2,2}^{2}$. Then, $\beta_{2,1}=\beta_{2,2}=\frac{1}{2}$. The upper bound of the rate loss of MS 1 becomes

$\Delta R_{1 u b}=\log _{2}\left[1+\frac{n_{t}}{2\left(n_{t}-1\right)}\left(\gamma_{1,1}^{2} \sin ^{2} \theta_{1,1}+\gamma_{1,2}^{2} \sin ^{2} \theta_{1,2}\right)\right]$,

which only depends on its receive SNRs and the per-cell CDI quantization errors.

When MS1 also locates near the cell edge, i.e., $\gamma_{1,1}^{2} \approx \gamma_{1,2}^{2}$, this MU-MIMO CoMP scenario is similar to the single cell MU-MIMO scenario. The rate loss depends on the receive SNR and the per-cell quantization errors, where the quantization errors of the local and cross channel contribute equally to the rate loss. Moreover, due to the fact that the SNRs of the local and cross links are low for the cell edge user MS1, the rate loss caused by the limited feedback is insignificant since this is a noise-limited scenario.

When MS1 moves from its cell edge to its cell center, the average energy of its local channel will increase much 
faster than the decrease of the average energy of its cross channel. Given the feedback bits of the local channel and the cross channel, it is shown from (11) that the rate loss caused by the limited feedback will increase dramatically. This means that the throughput of the cell center MS is severely limited by the quantization errors. Nonetheless, since $\gamma_{1,1}^{2} \gg \gamma_{1,2}^{2}$, the rate loss is mainly led by the quantization error of the local channel, while that of the cross channel has little contribution. This suggests that the rate loss can be reduced by allocating more bits to the quantization of local channel than to that of cross channel. Such a conclusion coincides with the observations obtained for SU-MIMO CoMP [9] and multi-user transmission using coordinated BF [11].

\section{B. When MS2 Locates at Cell Center}

As shown in Fig 2 (b), when MS 2 locates at the center of cell 2, we have $\alpha_{2,2}^{2} \gg \alpha_{2,1}^{2}$. As a result, $\beta_{2,1}$ approaches 0 while $\beta_{2,2}$ increases towards 1 .

When MS1 locates near the cell edge, the value of $\gamma_{1,2}$ approximately equals to that of $\gamma_{1,1}$. At this time, the contribution of the quantization error of the local channel is little because $\beta_{2,1} \approx 0$. The rate loss of MS1 is mainly caused by the quantization error of the cross channels. Since the increase of $\beta_{2,2}$ is less than the decrease of $\beta_{2,1}$, the rate loss of MS1 will be less when MS2 locates at cell center than that when MS2 locates at cell edge.

When MS1 locates near the cell center, we can observe from (10) that, when the receive SNR of its local channel is high, the impact of the quantization error of the local channel is less since $\beta_{2,1} \approx 0$. At the same time, the impact of the quantization error of the cross channel will be less as well since $\beta_{2,2} \leq 1$ and $\gamma_{1,2} \approx 0$. In general, the rate loss caused by limited feedback will be neglected. This implies that the feedback bits to both the local and the cross channels can be significantly reduced if the MSs locate at the cell center.

\section{Simulation RESUlts}

In this section, we verify our analysis through simulations. To be consistent with our analysis, we consider two BSs cooperatively serve two single antenna MSs. Each BS is equipped with 4 antennas. The cell radius $r$ is set to be $250 \mathrm{~m}$. Assume that the receive SNR of the cell edge MS, $\gamma_{e d g e}$, is $10 \mathrm{~dB}$. Consider the path loss factor $\epsilon$ as 3.76 , then the receive SNR of the signal from a BS to a MS with MS-BS distance of $d$ can be calculated according to $\gamma(d)=\gamma_{\text {edge }}+\epsilon 10 \log _{10}\left(\frac{d}{r}\right)$.

In the simulations, the small scale fading channels between BSs and MSs are independent and identical distributed Rayleigh channels. The codebooks for both the per-cell and the global channel quantization are obtained by the generalized Lloyd algorithm [14].

\section{A. Locations of MSs are Fixed}

Here we assume that two MSs locates on a straight line connecting the two BSs. Then the distance from MS1 to BS1 can represent the large scale fading factors of both local and cross channels of MS1. When MS1 moves from its cell edge to

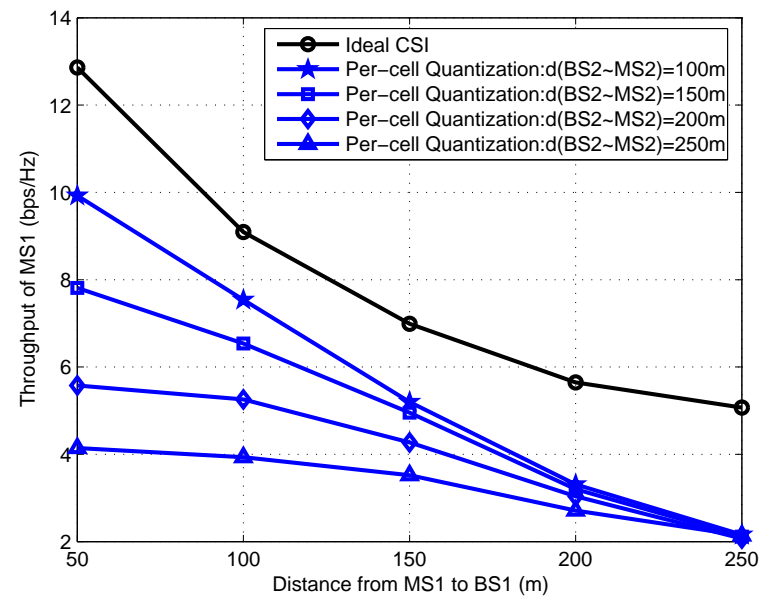

Fig. 3. Throughput of MS1 versus the distance from BS1 to MS1 when the distance from MS2 to BS2 are different.

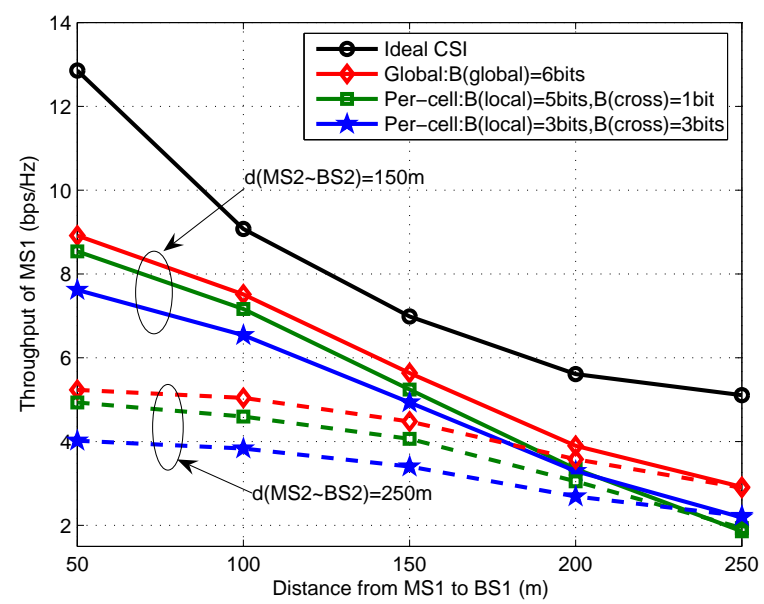

Fig. 4. Throughput of MS1 versus the distance from BS1 to MS1 using different quantization methods.

its cell center, the receive SNR of the local channel increases while that of the cross channel decreases.

The throughput of MS1 is obtained according to (4) by averaging over 1000 realizations of the small scale fading channel. In order to observe if our analysis still holds when the assumption of orthogonal MSs selection is not satisfied, MS2 is always served simultaneously together with MS1 no matter whether its quantized global channel is orthogonal to that of MS1 or not.

Figure 3 shows the average throughput of MS1 when the location of MS2 varies. Per-cell quantization is used and the feedback bits of both local and cross channels are fixed to be 3 bits. To observe the rate loss, the performance with ideal CSI is shown. Then the gap of the throughput using quantization from the throughput using ideal CSI reflects the rate loss. It is shown that when MS2 locates at cell edge, the average rate loss of MS1 increases when it moves from cell edge to cell 
center. Along with the movement of MS2 towards BS2, the rate loss of MS1 decreases. When MS2 locates at the cell center, the performance loss of MS1 even decreases when its local channel receive SNR increases. This agrees well will our theoretical analysis.

Figure 4 shows the throughput of MS1 using different quantization methods. Three quantization schemes are considered, which are the global channel quantization, the percell quantization with equal bits allocation and the per-cell quantization with unequal bits allocation. It is shown that the global quantization outperforms the per-cell quantization, and the performance of per-cell quantization can be improved by unequal bits allocation. These findings for the MU-MIMO CoMP coincide with those for coordinate BF [12]. However, we can observe that the impact of the position of MS2 on the throughput of MS1 is much larger than the quantization strategies.

\section{B. Locations of MSs are Randomly Distributed}

Now we simulate the case where the locations of two MSs are randomly distributed in two cells, and the two MSs are served with MU-MIMO CoMP by the two BSs. This corresponds to the scheme where a random user selection method is applied. The cumulative distribution function (CDF) of the throughput of MS1 is shown in Fig[5, which is obtained from 1000 random drops. The performances of the single cell MU-MIMO transmission with both ideal CSI and quantized CSI are also shown for comparison. To ensure the fairness of comparison with the CoMP scenario, two MSs are randomly distributed in a single cell and the BS is equipped with 8 antennas. Besides, we assume that the transmit power to the MSs are the same for both single cell and CoMP scenarios. This means that the BS transmit power of the single cell scenario is twice as much as per-BS transmit power of the CoMP scenario.

We can observe that the performance of the single cell MU-MIMO transmission degrades severely when 6 bits are used to quantize the $1 \times 8$ channel vectors. By contrast, the throughput gaps between the schemes using limited feedback and ideal CSI are not significant when only 6 bits are used to feed back two $1 \times 4$ channel vectors in the CoMP system. This is due to the fact that, for MU-MIMO CoMP, in a large probability the average channel energy of the local channel for a randomly distributed MS is higher than that of its cross channel. Therefore, the impact of quantization error on the rate loss will be largely mitigated according to our analysis in Section III]

\section{CONCLUSION}

In this paper, we investigated the impact of channel asymmetry on CoMP MU-MIMO systems with limited feedback based on per-cell quantization strategy. Our analysis showed that the rate loss of a MS caused by limited feedback is dependent on the receive SNR, the number of transmit antennas, the CSI quantization error, as well as the location of its paired MSs in the cooperative cells. For a cell center MS, the position

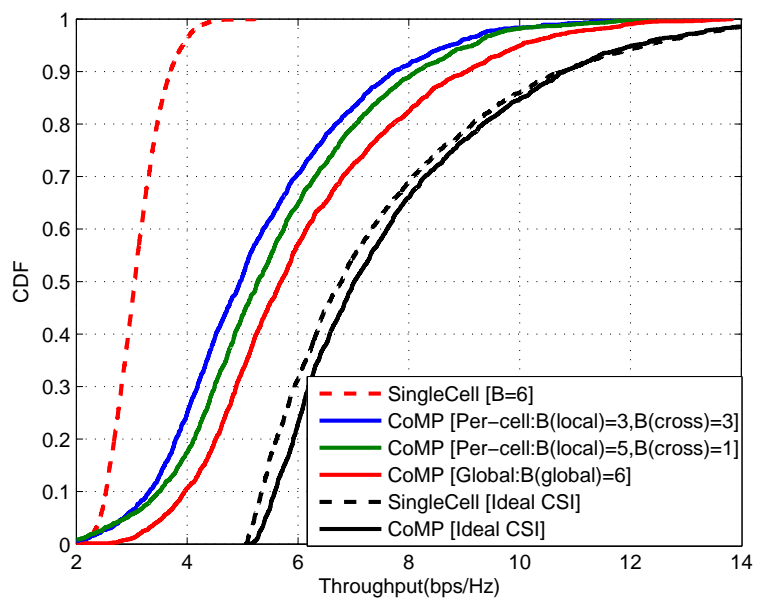

Fig. 5. CDF of per MS throughput when the two MSs are randomly distributed. The impact of the limited feedback on both single cell MU-MIMO and MU-MIMO CoMP systems are compared.

of its paired MSs is the dominate factor of its performance loss. When the paired MSs locates at cell edge, the throughput of the cell center MS will be interference limited. When the paired MSs also locates at cell center, the rate loss induced by the quantization error is neglectable. On the other hand, for a cell edge MS, its rate loss when its paired MSs locates at cell center will also be less than that when the paired MSs locates at cell edge.

\section{APPENDiX A}

DERIVATION OF THE UPPER BOUND OF $\Delta R_{k}$

Applying Jensen's inequality to (8), we can obtain the following upper bound of the rate loss of MS $k$,

$$
\begin{aligned}
\Delta R_{k} & <\log _{2}\left[1+\frac{P}{\sigma_{n}^{2}} \mathbb{E}\left\{\sum_{j \neq k} \frac{1}{\left\|\hat{\mathbf{g}}_{j}\right\|^{2}}\left|\mathbf{g}_{k} \hat{\mathbf{g}}_{j}^{H}\right|^{2}\right\}\right] \\
& <\log _{2}\left[1+\frac{P}{\sigma_{n}^{2}} \sum_{j \neq k} \mathbb{E}\left\{\frac{1}{\left\|\hat{\mathbf{g}}_{j}\right\|^{2}}\right\} \mathbb{E}\left\{|\underbrace{\mathbf{g}_{k} \hat{\mathbf{g}}_{j}^{H}}_{Q_{j, b}}|^{2}\right\}\right] .
\end{aligned}
$$

Let us first find the upper bound of $\mathbb{E}\left\{\frac{1}{\left\|\hat{\mathbf{s}}_{j}\right\|^{2}}\right\}$ in (12) as follows. Again by Jensen's inequality, we have

$$
\mathbb{E}\left\{\frac{1}{\left\|\hat{\mathbf{g}}_{j}\right\|^{2}}\right\}<\frac{1}{\sum_{b=1}^{N} \mathbb{E}\left\{\rho_{j, b}^{2}\right\}}=\frac{1}{n_{t} \sum_{b=1}^{N} \alpha_{j, b}^{2}} .
$$

Then before finding the upper bound of $\mathbb{E}\left\{\left|Q_{j, b}\right|^{2}\right\}$ in (12), we expand the expression of $Q_{j, b}$. According to [5], the $\mathbf{h}_{k, b}$ can be expressed as $\mathbf{h}_{k, b}=\cos \theta_{k, b} \hat{\mathbf{h}}_{k, b}+\sin \theta_{k, b} \mathbf{s}_{k, b}$, where $\mathbf{s}_{k, b}$ is isotropic distributed in the nullspace of $\hat{\mathbf{h}}_{k, b}$. Then the expression of $Q_{j, b}$ can be obtained as follows,

$Q_{j, b}=\sum_{b=1}^{N} \varepsilon_{b} \cos \theta_{k, b} \hat{\mathbf{h}}_{k, b} \hat{\mathbf{h}}_{j, b}^{H}+\sum_{b=1}^{N} \varepsilon_{b} \sin \theta_{k, b} \mathbf{s}_{k, b} \hat{\mathbf{h}}_{j, b}^{H}$,

where $\varepsilon_{b}=\rho_{k, b} \rho_{j, b}$. 
Then we have

$$
\begin{aligned}
\mathbb{E}\left\{\left|Q_{j, b}\right|^{2}\right\}= & \mathbb{E}\left\{\sum_{b=1}^{N} \varepsilon_{b}^{2} \cos ^{2} \theta_{k, b}\left|\hat{\mathbf{h}}_{k, b} \hat{\mathbf{h}}_{j, b}^{H}\right|^{2}\right\} \\
& +\mathbb{E}\left\{\sum_{b=1}^{N} \varepsilon_{b}^{2} \sin ^{2} \theta_{k, b}\left|\mathbf{s}_{k, b} \hat{\mathbf{h}}_{j, b}^{H}\right|^{2}\right\} \\
< & \mathbb{E}\left\{\sum_{b=1}^{N} \varepsilon_{b}^{2}\left|\hat{\mathbf{h}}_{k, b} \hat{\mathbf{h}}_{j, b}^{H}\right|^{2}\right\} \\
& +\mathbb{E}\left\{\sum_{b=1}^{N} \varepsilon_{b}^{2} \sin ^{2} \theta_{k, b}\left|\mathbf{s}_{k, b} \hat{\mathbf{h}}_{j, b}^{H}\right|^{2}\right\} .
\end{aligned}
$$

Since we assume that the scheduled MSs are mutually orthogonal in terms of quantized global CDI, we have

$$
\hat{\mathbf{g}}_{k} \hat{\mathbf{g}}_{j}^{H}=\sum_{b=1}^{N} \varepsilon_{b} \hat{\mathbf{h}}_{k, b} \hat{\mathbf{h}}_{j, b}^{H}=0,
$$

where $\hat{\mathrm{g}}_{k}$ is the quantized global CDI of MS $k$. Then by assuming that the channels from one BS to multiple MSs are independent, we have

$$
\mathbb{E}\left\{\left|\hat{\mathbf{g}}_{k} \hat{\mathbf{g}}_{j}^{H}\right|^{2}\right\}=\sum_{b=1}^{N} \mathbb{E}\left\{\varepsilon_{b}^{2}\left|\hat{\mathbf{h}}_{k, b} \hat{\mathbf{h}}_{j, b}^{H}\right|^{2}\right\}=0 .
$$

Substituting (15) into (14) we can get

$$
\begin{aligned}
\mathbb{E}\left\{\left|Q_{j, b}\right|^{2}\right\} & <\sum_{b=1}^{N} \sin ^{2} \theta_{k, b} \mathbb{E}\left\{\varepsilon_{b}^{2}\right\} \mathbb{E}\left\{\left|\mathbf{s}_{k, b} \hat{\mathbf{h}}_{j, b}^{H}\right|^{2}\right\} \\
& =\frac{1}{n_{t}-1} \sum_{b=1}^{N} \alpha_{k, b}^{2} \alpha_{j, b}^{2} \sin ^{2} \theta_{k, b},
\end{aligned}
$$

where $\mathbb{E}\left\{\left|\mathbf{s}_{k, b} \hat{\mathbf{h}}_{j, b}^{H}\right|^{2}\right\}=\frac{1}{n_{t}-1}$ is obtained according to [5].

Substituting (13) and (16) into (12), we can obtain the upper bound of the rate loss as in (9).

\section{REFERENCES}

[1] M. K. Karakayali, G. J. Foschini, and R. A. Valenzuela, "Network coordination for spectrally efficient communications in cellular systems," IEEE Wireless Commun. Mag., vol. 13, pp. 56-61, 2006.

[2] A. Tölli and M. Codreanu and M. Juntti, "Cooperative MIMO-OFDM cellular system with soft handover between distributed base station antennas," IEEE Trans. Wireless Commun., vol. 7, pp. 1428-1440, Apr. 2008.

[3] H. Huang, M. Trivellato, A. Hottinen, M. Shafi, P. Smith, and R. A. Valenzuela, "Increasing downlink cellular throughput with limited network MIMO coordination," IEEE Trans. Wireless Commun., vol. 8, pp. 2983 - 2989, Jun. 2009.

[4] D. Love, R. W. Heath Jr., V. K. N. Lau, D. Gesbert, B. D. Rao, and M. Andrews, "An overview of limited feedback in wireless communication systems," IEEE J. Sel. Areas Commun., vol. 26, pp. 1341-1365, Oct. 2008.

[5] N. Jindal, "MIMO broadcast channels with finite rate feedback," IEEE Trans. Inf. Theory, vol. 52, no. 11, pp. 5045-5059, 2006.

[6] T. Yoo, N. Jindal, and A. Goldsmith, "Multi-antenna downlink channels with limited feedback and user selection," IEEE J. Sel. Areas Commun., vol. 25 , no. 7, pp. 1478-1491, 2007.

[7] W. Choi and J. G. Andrews, "The capacity gain from intercell scheduling in multi-antenna systems," IEEE Trans. Wireless Commun., vol. 7, pp. 714-725, Feb. 2008.

[8] S. Han, C. Yang, M. Bengtsson, and A. I. Perez-Neira, "Channel norm based user scheduling in coordinated multi-point systems," in Proc. IEEE Glob. Telecom. Conf. (GlobeCom), 2009.

[9] C. K. A. Yeung and S. Sanayei, "Enhanced trellis based vector quantization for coordinated beamforming," in Proc. IEEE Int. Conf. Acoust., Speech and Sig. Proc. (ICASSP), 2010.

[10] D. Su and C. Yang, "Low feedback codebook for CoMP SU-MIMO systems," in Chinese patent pending, No. 201010127347.4, Mar. 2010.
[11] R. Bhagavatula and R. W. Heath Jr., "Multicell cooperative beamforming to maximize sum-rate with limited feedback," submitted to IEEE Trans. Sig. Proc., 2009.

[12] R. Bhagavatula, R. W. Heath Jr., and B. Rao, "Limited feedback with joint CSI quantization for multicell cooperative generalized eigenvector beamforming," in Proc. IEEE Int. Conf. Acoust., Speech and Sig. Proc. (ICASSP), 2010.

[13] T. Yoo and A. Goldsmith, "On the optimality of multiantenna broadcast scheduling using zero-forcing beamforming," IEEE J. Sel. Areas Commun., vol. 24, no. 3, p. 528C541, 2006.

[14] A. Gersho and R. M. Gray, Vector Quantization and Signal Compression. Kluwer Academic Publishers, 1993. 